\title{
A preliminary study of synthetic magnetic resonance imaging in rectal cancer: imaging quality and preoperative assessment
}

\author{
Li Zhao ${ }^{1}$, Meng Liang ${ }^{1}$, Pu-yeh Wur ${ }^{2}$, Yang Yang ${ }^{1}$, Hongmei Zhang ${ }^{1 *}$ and Xinming Zhao ${ }^{1 *}$ (])
}

\begin{abstract}
Purpose: To compare the imaging quality, T stage and extramural venous invasion (EMVI) evaluation between the conventional and synthetic T2-weighted imaging (T2WI), and to investigate the role of quantitative values obtained from synthetic magnetic resonance imaging (MRI) for assessing nodal staging in rectal cancer (RC).

Methods: Ninety-four patients with pathologically proven RC who underwent rectal MRI examinations including synthetic MRI were retrospectively recruited. The image quality of conventional and synthetic T2WI was compared regarding signal-to-noise ratio (SNR), contrast-to-noise (CNR), sharpness of the lesion edge, lesion conspicuity, absence of motion artifacts, and overall image quality. The accuracy of T stage and EMVI evaluation on conventional and synthetic T2WI were compared using the Mc-Nemar test. The quantitative T1, T2, and PD values were used to predict the nodal staging of MRI-evaluated node-negative RC.

Results: There were no statistically significant differences between conventional and synthetic T2WI in SNR, CNR, overall image quality, lesion conspicuity, and absence of motion artifacts $(p=0.058-0.978)$. There were no significant differences in the diagnostic accuracy of T stage and EMVI between conventional and synthetic T2WI from two observers ( $p=0.375$ and 0.625 for T stage; $p=0.625$ and 0.219 for EMVI). The T2 value showed good diagnostic performance for predicting the nodal staging of $\mathrm{RC}$ with the area under the receiver operating characteristic, sensitivity, specificity, and accuracy of $0.854,90.0 \%, 71.4 \%$, and $80.3 \%$, respectively.
\end{abstract}

Conclusions: Synthetic MRI may facilitate preoperative staging and EMVI evaluation of RC by providing synthetic T2Wl and quantitative maps in one acquisition.

Keywords: Rectal cancer, Magnetic resonance imaging, Synthetic imaging, Evaluation study

\section{Key Points}

- Synthetic T2WI provides comparable image quality with the conventional T2WI.

- Synthetic T2WI enables similar diagnostic accuracy in local staging of rectal cancer.

\footnotetext{
*Correspondence: 13581968865@163.com; zhaoxinming@cicams.ac.cn 1 Department of Diagnostic Radiology, National Cancer Center/ National Clinical Research Center for Cancer/Cancer Hospital, Chinese Academy of Medical Sciences and Peking Union Medical College, No. 17, Panjiayuan Nanli, Chaoyang District, Beijing 100021, China

Full list of author information is available at the end of the article
}

- Synthetic MRI facilitate preoperative evaluation of rectal cancer by providing multiple images.

\begin{abstract}
Introduction
Colorectal cancer is the third most common malignancy worldwide, ranking third in mortality among female and male, respectively [1]. Rectal cancer (RC) accounts for approximately $30 \%-35 \%$ of colorectal cancer cases, which are mostly adenocarcinoma [2]. Magnetic resonance imaging (MRI), especially high-resolution T2-weighted imaging (T2WI), is recommended to be routinely used
\end{abstract}


for assessing $\mathrm{RC}$ local stage, which is critical for treatment decisions and the prognosis prediction [3, 4].

Besides the conventional contrast-weighted imaging, quantitative relaxation mappings have been shown to play a certain role in identifying the tumor grade, metastatic lymph nodes, lymphovascular invasion, and therapeutic response of several tumors [5-9]. However, the separate acquisitions of quantitative mappings and contrast-weighted images can be time-consuming. Synthetic MRI, in which a multi-echo and multi-delay acquisition scheme is adopted, can be advantageous of shorting scanning time by simultaneously quantifying $\mathrm{T} 1, \mathrm{~T} 2$ and proton density (PD) relaxometry and generating synthetic contrast-weighted images in a single scan $[10,11]$.

Synthetic MRI has been demonstrated to have excellent correlation with conventional mapping methods and comparable image quality to that of conventional contrast-weighted images in brain and knee [12-14]. Additionally, there have been some promising findings of synthetic MRI in various tumors, such as prostate cancer, breast cancer, bone metastasis $[6,15,16]$. Our previous study has proven that quantitative T1 and T2 values generated from synthetic MRI were useful for predicting prognostic factors of RC [17]. Another study showed that radiomics model based on synthetic MRI could improve the diagnostic performance of extramural venous invasion (EMVI) in rectal cancer [18]. To the best of our knowledge, the feasibility of the contrast-weighted imaging generated from synthetic MRI has not yet been reported.

Recently, the diagnostic accuracy and interobserver agreement of conventional nodal staging in RC remain unsatisfactory [19]. A previous study suggested that $\mathrm{T} 2$ value may be useful in differentiating metastatic lymph nodes in RC, but is limited by the node-by-node approach to match the lymph node on MRI with the pathological specimen [9]. Although previous studies have confirmed the associations between the characteristics of the primary tumor and the nodal staging, few studies have focused on the MRI-evaluated node-negative RC.

The aim of this study was to compare the image quality, $\mathrm{T}$ stage, and EMVI assessment between the conventional and synthetic T2WI, and to investigate the quantitative values for a more accurate nodal staging of MRI-evaluated node-negative RC.

\section{Materials and methods Participants}

Our Institutional Review Board approved this retrospective single-center study and the informed consent was waived. In total, 143 patients with pathologically confirmed rectal adenocarcinoma who underwent rectal
MRI examinations including the synthetic MRI sequence between November 2018 and February 2020 were enrolled. Exclusion criteria were as follows: (1) those who received neoadjuvant treatment before the surgery; (2) time interval between the MRI examination and surgery greater than 4 weeks. Finally, 94 patients were included in the study. Of these, 18 participants with suspicious metastatic lymph node on preoperative MRI were excluded from quantitative evaluation of nodal staging. The flowchart of the study cohort is shown in Fig. 1.

Pathological characteristics of surgical specimens were assessed according to the eighth edition of the American Joint Committee on Cancer (AJCC) TNM staging system [20].

\section{MRI acquisition}

All MRI examinations were performed on a $3.0 \mathrm{~T}$ scanner (SIGNA Pioneer, GE Healthcare, Milwaukee, WI) equipped with a 32-channel phased-array body coil. For bowel preparation, glycerin enema was used to empty the feces before the examination. Anisodamine hydrochloride (10 mg, except those with contraindications) was intramuscularly injected $15 \mathrm{~min}$ before the examination to reduce peristaltic artifacts. Axial T1-weighted imaging (T1WI), diffusion-weighted imaging (DWI) (b values of 0 and $1000 \mathrm{~s} / \mathrm{mm}^{2}$ ), oblique axial/coronal (perpendicular/parallel to the maximum tumor length), sagittal T2WI, synthetic MRI, and dynamic contrast-enhanced sequence were obtained. Synthetic MRI was performed before the injection of contrast agent in the oblique axial plane, using a QRAPMASTER (quantification of relaxation times and proton density by multiecho acquisition of a saturation-recovery using turbo spin-echo readout) sequence with two echo times $(19.5 / 97.3 \mathrm{~ms})$ and four saturation delay times $(210 / 610 / 1810 / 3810 \mathrm{~ms})$. The detailed acquisition parameters are listed in Table 1. Raw data of synthetic MRI were loaded into SyMRI 8.0 (SyntheticMR, Linköping, Sweden) for postprocessing, and quantitative T1, T2, PD maps and synthetic contrastweighted images were automatically generated within $10 \mathrm{~s}$ (Fig. 2).

\section{Subjective image quality analysis}

The subjective image quality of conventional and synthetic T2WI was evaluated by two radiologists with 5 and 8 years of experience in rectal imaging, who were blinded to pathological information and acquisition methods. The observers scored the image quality based on following 4 factors on a 5 -point-Likert scale: (1) Sharpness of the lesion edge $(1=$ not sharp; $2=$ a little sharp; $3=$ moderately sharp; $4=$ well sharp; $5=$ very sharp); (2) Lesion conspicuity $(1=$ difficult to find; $2=$ minimally perceivable; $3=$ recognizable; $4=$ easy 
143 patients with rectal cancer who underwent preoperative rectal MRI examination including synthetic

MRI between November 2018 and February 2020

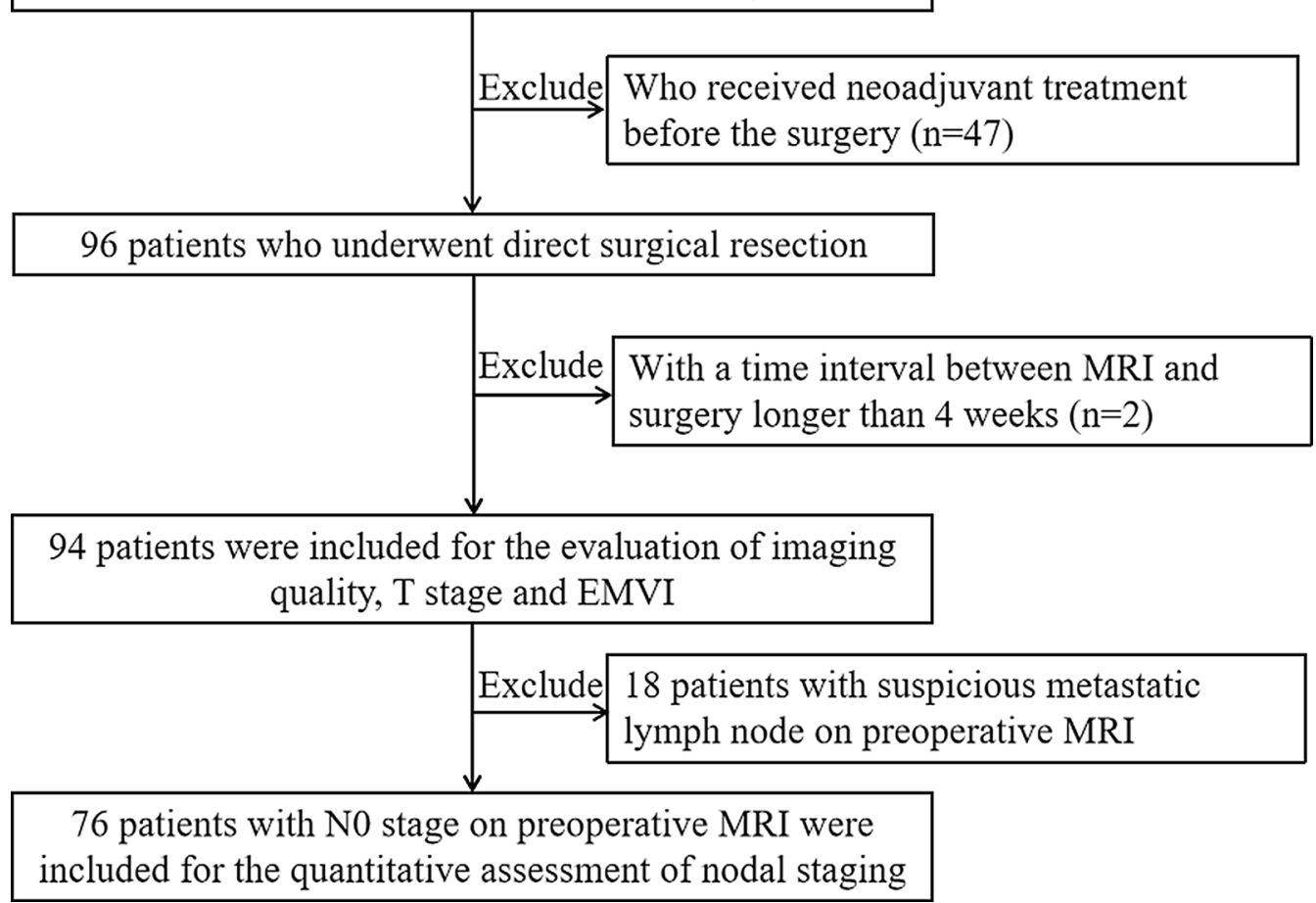

Fig. 1 Flow chart of the study cohort

Table 1 MRI parameters

\begin{tabular}{llllll}
\hline & T1WI & T2WI & Synthetic MRI & DWI & DCE \\
\hline Sequence & FSE & FSE & QRAPMASTER & SS-EPI & DISCO \\
Imaging plane & Axial & Oblique axial, oblique coronal, sagittal & Oblique axial & Axial & Axial \\
Repetition time (ms) & 588 & $5115,5912,5062$ & 4000 & 4574 & 4.8 \\
Echo time (ms) & Min full & $85.0,98.8,90.0$ & $19.5 / 97.3$ & Minimum & 2.0 \\
Slice thickness/gap (mm) & $5 / 1$ & $3 / 0.3$ & $3 / 0.3$ & $5 / 1$ & $2 / 0$ \\
Field of view (cm) & $40 \times 40$ & $18 \times 18,24 \times 24,24 \times 24$ & $24 \times 24$ & $40 \times 40$ & $36 \times 36$ \\
Matrix & $320 \times 256$ & $320 \times 224,320 \times 256,320 \times 224$ & $320 \times 256$ & $128 \times 128$ & $288 \times 192$ \\
Echo train length & 3 & $24,32,24$ & 16 & - & - \\
Bandwidth (kHz) & 50.00 & $50.00,63.50,50.00$ & 27.78 & 250 & 142.86 \\
Acquisition time (min: s) & $0: 57$ & $02: 39,02: 40,2: 37$ & $04: 32$ & $01: 36$ & Per phase 0:09
\end{tabular}

MRI, magnetic resonance imaging; T1WI, T1-weighted imaging; T2WI, T2-weighted imaging; DWI, diffusion-weighted imaging; DCE, dynamic contrast enhanced; FSE, fast spin-echo; QRAPMASTER, quantification of relaxation times and proton density by multiecho acquisition of a saturation recovery using turbo spin-echo readout; SS-EPI, single-shot echo-planar imaging; DISCO, differential sub-sampling with cartesian ordering

to detect, good contrast of lesion; $5=$ excellent contrast of lesion); (3) Motion artifacts ( $1=$ severe, difficult to diagnose; $2=$ a little severe, accessible to diagnose; $3=$ moderate; $4=$ mild; $5=$ absence of artifacts); (4) Overall image quality (the three factors above added together, $1=$ unacceptable; $2=$ poor; $3=$ moderate; $4=$ good; $5=$ excellent). The patient order was randomized, as was the review order of the conventional or synthetic T2WI. 

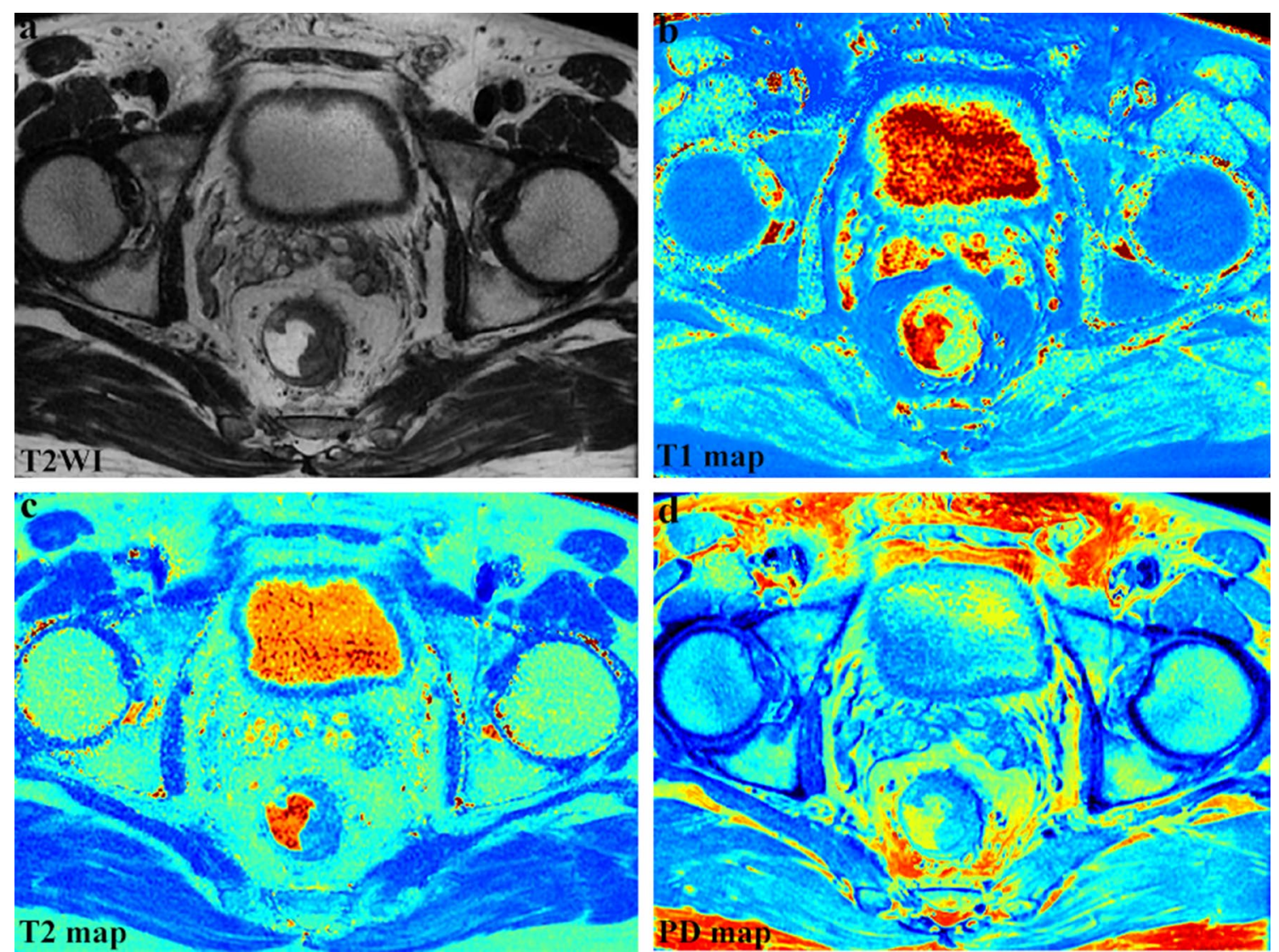

Fig. 2 Representative synthetic magnetic resonance images from a 57-year-old male patient with rectal cancer. (a-d) T2-weighted imaging (T2WI), T1 map, T2 map, and PD map

\section{Objective image quality analysis}

Regions of interest (ROI) were manually drawn on conventional oblique axial T2WI and synthetic T2WI using ITK-SNAP software (version 2.2.0, www.itksnap.org) by the same two radiologists. The ROI for the tumor was delineated on each slice along the margin of the tumor, resulting in a $3 \mathrm{D}$ whole tumor ROI. The normal tissue ROI was drawn in homogeneous normal rectum tissue distant from the tumor area, including the entire rectal wall at a single slice. The background ROI was a circular area with a diameter of $1 \mathrm{~cm}$, placed within the field of view but outside the body surface. The mean and standard deviation of the signal intensity were obtained from each ROI. Signal-to-noise ratio (SNR) and contrast-tonoise (CNR) were calculated based on following formulas [21]: the background noise, and $S_{\text {tissue }}$ denotes the mean signal intensity of the normal tissue (Fig. 3).

\section{Preoperative evaluation of RC}

The same two radiologists independently assessed the $\mathrm{T}$ stage and EMVI status on conventional and synthetic T2WI. The T stage was evaluated based on the European Society for Medical Oncology guidelines [3]. In addition, EMVI status was assessed based on a 5-point scoring system proposed by Smith et al. [22]: $(0=$ definitely absent; $1=$ probably absent; $2=$ indeterminate; $3=$ probably present; $4=$ definitely present). Scores of 3 or 4 were regarded as positive EMVI. Criteria for malignant node was according to the European Society of Gastrointestinal and Abdominal Radiology recommendations [23]: (1) short axis diameter $\geq 9 \mathrm{~mm}$; (2) short axis

$$
\mathrm{SNR}=S_{\text {tumor }} / \mathrm{SD}_{\text {background }}, \mathrm{CNR}=\left|S_{\text {tumor }}-S_{\text {tissue }}\right| / / \mathrm{SD}_{\text {background }}
$$

where $S_{\text {tumor }}$ is the mean signal intensity within the tumor, $\mathrm{SD}_{\text {background }}$ represents the standard deviation of diameter $5-8 \mathrm{~mm}$ and $\geq 2$ morphologically suspicious characteristics; (3) short axis diameter $<5 \mathrm{~mm}$ and 3 

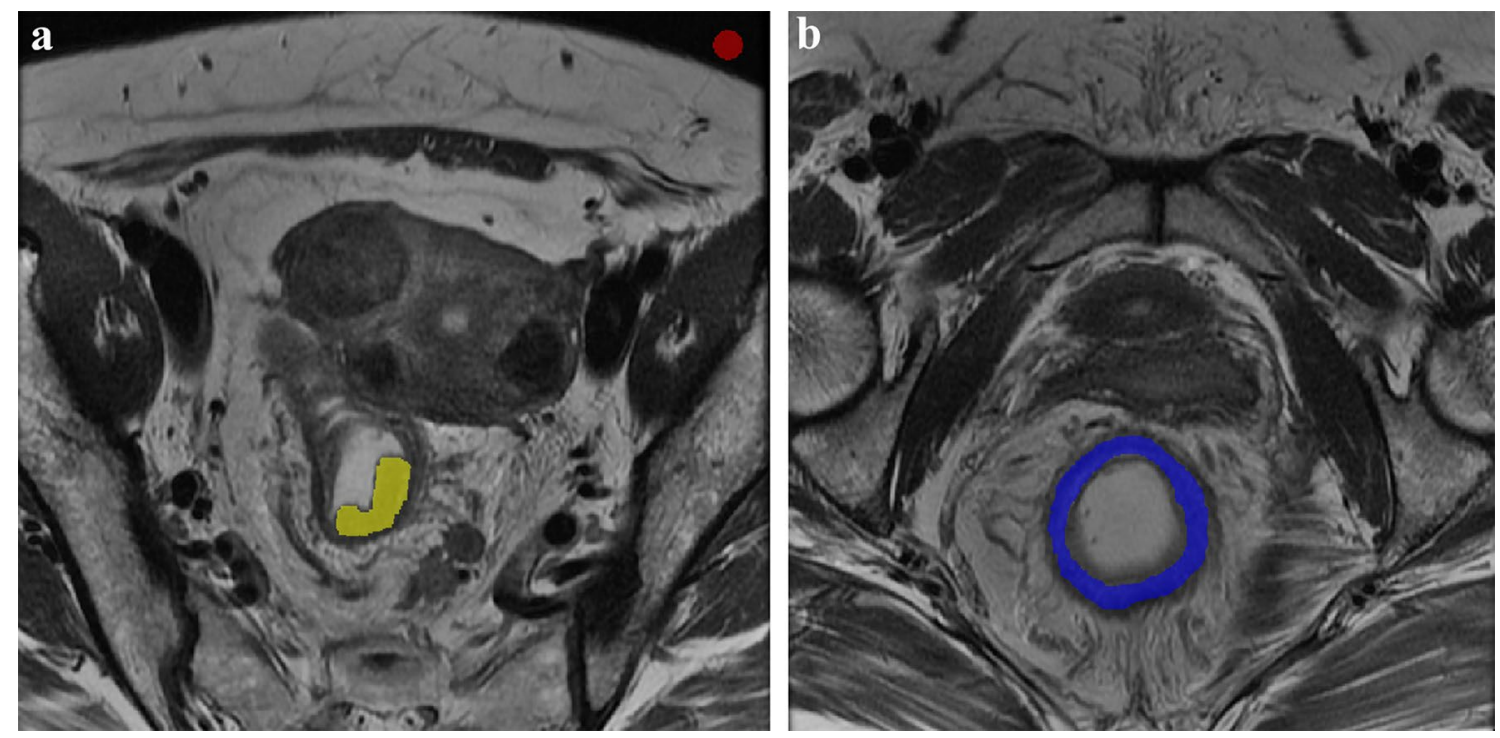

Fig. 3 Signal-to-noise ratio (SNR) and contrast-to-noise (CNR) were calculated based on three regions of interest (ROIs). The yellow ROI represents the tumor, which was delineated slice by slice along the tumor margin. The red ROI on background was placed within the field of view but outside the body surface. The blue ROI represents normal tissue, which was drawn in normal rectum tissue at a single slice. The mean and standard deviation of the signal intensity were obtained from each ROI to calculate SNR and CNR

morphologically suspicious characteristics; (4) all mucinous lymph nodes (any size). Morphologically suspicious criteria included round shape, irregular border, and heterogeneous signal. If there was any obvious discordance of nodal staging between two radiologists, a senior radiologist with 21 years of experience in rectal imaging made the final decision.

\section{Quantitative assessment}

The whole tumor ROIs drawn on synthetic T2WI by two radiologists above were transferred to the quantitative maps using ITK-SNAP software. The mean T1, T2, and PD values were automatically obtained. Quantitative values acquired by the senior radiologist were used to assess the nodal staging of MRI-evaluated node-negative RC.

\section{Statistical analysis}

The inter-observer variability for image quality score and the tumor evaluation was assessed using kappa statistics (0.21-0.40, fair; 0.41-0.60, moderate; 0.61-0.80, good; 0.81-1.00, excellent). The intraclass correlation coefficient (ICC) was used to investigate the interobserver agreement of the SNR, CNR and quantitative values $(0.21-0.40$, fair; $0.41-0.60$, moderate; $0.61-0.80$, good; 0.81-1.00, excellent) [5]. Continuous variables were compared using the independent samples $t$ test or the Mann-Whitney $U$ test according to the normality of data distribution. The Wilcoxon signed-rank test was adopted to compare the image quality scores, SNR and
CNR between conventional and synthetic T2WI. With pathological results as the reference standard, the differences between conventional and synthetic T2WI in evaluating the $\mathrm{T}$ stage and EMVI were determined using the Mc-Nemar test. Receiver operating characteristic (ROC) curves were used to assess diagnostic performance of quantitative values in nodal staging. A two-sided $p$ value $<0.05$ was considered statistically significant. All statistical analyses were performed using SPSS 20.0 (IBM, Armonk, NY) and MedCalc 11.4 (MedCalc, Mariakerke, Belgium).

\section{Results \\ Clinical characteristics}

All 94 patients received radical surgical resection with pathological negative circumferential resection margin. The clinical characteristics of the patients are summarized in Table 2.

\section{Image quality analysis}

The image quality scores of conventional and synthetic T2WI are shown in Table 3. There were no statistically significant differences between conventional and synthetic T2WI in SNR, CNR, overall image quality, lesion conspicuity, and absence of motion artifacts $(p=0.058$ 0.978 for all comparison pairs, Table 3 ). Regarding the sharpness of the lesion edge, one observer's evaluation showed that there was no significant difference between the conventional and synthetic T2WI $(p=0.127)$, while 
Table 2 Clinical characteristics

\begin{tabular}{|c|c|}
\hline Characteristics & Number of patients \\
\hline Mean age, years (range) & $57(36-76)$ \\
\hline \multicolumn{2}{|l|}{ Gender } \\
\hline Male & 66 \\
\hline Female & 28 \\
\hline \multicolumn{2}{|l|}{ Surgical resection type } \\
\hline Low anterior or anterior resection & 56 \\
\hline Abdominoperineal resection & 33 \\
\hline Hartman's resection & 5 \\
\hline \multicolumn{2}{|l|}{ Differentiation } \\
\hline Well/Moderate & 59 \\
\hline Poor & 35 \\
\hline \multicolumn{2}{|l|}{ pT stage } \\
\hline $\mathrm{T} 1$ & 6 \\
\hline $\mathrm{T} 2$ & 33 \\
\hline T3 & 44 \\
\hline T4 & 11 \\
\hline \multicolumn{2}{|l|}{ pNstage } \\
\hline No & 59 \\
\hline N1 & 20 \\
\hline N2 & 15 \\
\hline \multicolumn{2}{|l|}{ pEMVI } \\
\hline Absent & 71 \\
\hline Present & 23 \\
\hline \multicolumn{2}{|l|}{ Tumor location } \\
\hline Upper & 14 \\
\hline Middle & 44 \\
\hline Lower & 36 \\
\hline
\end{tabular}

pEMVI, pathological extramural venous invasion

the other observer's evaluation had a significant difference $(p=0.018)$. Representative images from one patient are shown in Fig. 4.

\section{Evaluation of T stage and EMVI}

Comparisons of the diagnostic accuracy of $\mathrm{T}$ stage and EMVI using conventional and synthetic T2WI are presented in Table 4. With pathological $\mathrm{T}$ stage as the reference standard, there were no significant differences in the diagnostic accuracy of $\mathrm{T}$ stage between conventional and synthetic T2WI from both observers $(p=0.375$ and 0.625). Similarly, the differences in the accuracy for evaluating EMVI between conventional and synthetic T2WI were not statistically significant from both observers $(p=0.625$ and 0.219$)$.

\section{Quantitative assessment}

Among the 76 patients without suspicious metastatic lymph node on preoperative MRI, 56 were pathologically confirmed pN0 stage, and 20 were pN1-2. The differences in the T1, T2 and PD values between the pN0 and pN1-2 groups are listed in Table 5. The T2 values of the pN1-2 group were significantly lower than those of the $\mathrm{pNO}$ group $(p<0.001)$. The T2 value demonstrated good diagnostic performance for predicting nodal staging of preoperatively node-negative $\mathrm{RC}$ with the area under the ROC (AUC), sensitivity, specificity, and accuracy of 0.854 ( $95 \%$ confidence interval, CI, $0.755-0.925), 90.0 \%, 71.4 \%$, and 80.3\%, respectively. The ROC curve is shown in Fig. 5.

\section{Interobserver reliability}

The results of interobserver agreements are summarized in Additional file 1: Table S1. The SNR and CNR of conventional and synthetic T2WI all demonstrated excellent interobserver agreements (ICC $>0.9$ ). The interobserver agreements of subjective image quality scores for conventional and synthetic T2WI were good or excellent (kappa values $=0.800-0.957$ ). The interobserver agreements of mrT stage and mrEMVI were all excellent for conventional and synthetic T2WI (kappa values $=0.892$ and 0.810 for mrT stage; 0.854 and 0.865 for mrEMVI). The T1, T2, and PD value demonstrated excellent interobserver agreement $(\mathrm{ICC}=0.916,0.953$, and 0.973, respectively).

\section{Discussion}

We preliminarily investigated the feasibility of using synthetic MRI for the preoperative evaluation of RC. Synthetic T2WI provides similar image quality with the conventional T2WI, and achieves comparable diagnostic accuracy in assessing $\mathrm{T}$ stage and EMVI in RC patients. Additionally, T2 value demonstrated good diagnostic performance of nodal staging of MRI-evaluated nodenegative patients.

The main advantage of synthetic MRI is the simultaneous generation of multiple images in a single scan, including quantitative relaxation mappings and synthetic morphological contrast-weighted images. The efficiency of synthetic MRI in the clinical application may depend on whether the total acquisition time is reduced and image quality is favorable. The acquisition time of synthetic MRI in our study was similar to that of T2 mapping in previous studies $[9,24]$, while synthetic MRI could simultaneously provide information of $\mathrm{T} 1$ mapping, $\mathrm{PD}$ mapping, T2WI, etc. Therefore, the scanning time of synthetic MRI is less than that with separately acquired contrast-weighted images and quantitative relaxation mappings.

Another primary issue is to ensure the image quality of synthetic MRI for further extensive clinical application. Our results demonstrated that the image quality scores of synthetic T2WI were comparable to that of conventional T2WI in terms of the SNR, CNR, overall 
Table 3 Comparison of image quality between conventional and synthetic T2WI

\begin{tabular}{|c|c|c|c|}
\hline & Conventional $\mathrm{T}_{2} \mathrm{WI}$ & Synthetic $\mathrm{T}_{2} \mathrm{WI}$ & $P$ value \\
\hline \multicolumn{4}{|l|}{ SNR } \\
\hline Observer 1 & $29.70 \pm 12.17$ & $26.85(18.33,41.24)$ & 0.978 \\
\hline Observer 2 & $29.14 \pm 11.94$ & $25.85(19.11,41.06)$ & 0.717 \\
\hline \multicolumn{4}{|l|}{ CNR } \\
\hline Observer 1 & $5.41(2.00,9.67)$ & $5.66(2.89,11.71)$ & 0.145 \\
\hline Observer 2 & $4.99(2.39,9.37)$ & $5.27(3.10,11.84)$ & 0.058 \\
\hline \multicolumn{4}{|c|}{ Overall image quality } \\
\hline Observer 1 & $4.63 \pm 0.53$ & $4.60 \pm 0.54$ & 0.592 \\
\hline Observer 2 & $4.64 \pm 0.51$ & $4.57 \pm 0.54$ & 0.239 \\
\hline \multicolumn{4}{|c|}{ Lesion conspicuity } \\
\hline Observer 1 & $4.69 \pm 0.53$ & $4.71 \pm 0.52$ & 0.617 \\
\hline Observer 2 & $4.65 \pm 0.56$ & $4.66 \pm 0.54$ & 0.808 \\
\hline \multicolumn{4}{|c|}{ Sharpness of the lesion edge } \\
\hline Observer 1 & $4.59 \pm 0.50$ & $4.69 \pm 0.46$ & $0.018^{*}$ \\
\hline Observer 2 & $4.64 \pm 0.51$ & $4.71 \pm 0.46$ & 0.127 \\
\hline \multicolumn{4}{|c|}{ Absence of motion artifacts } \\
\hline Observer 1 & $4.68 \pm 0.53$ & $4.56 \pm 0.58$ & 0.069 \\
\hline Observer 2 & $4.64 \pm 0.55$ & $4.53 \pm 0.58$ & 0.112 \\
\hline
\end{tabular}

SNR and CNR following the normal distribution are expressed as mean \pm standard deviation; otherwise, expressed as median (first quartile, third quartile). The subject image scores are expressed as means \pm standard deviations SNR, signal-to-noise ratio; CNR, contrast-to-noise ratio ${ }^{*} p<0.05$
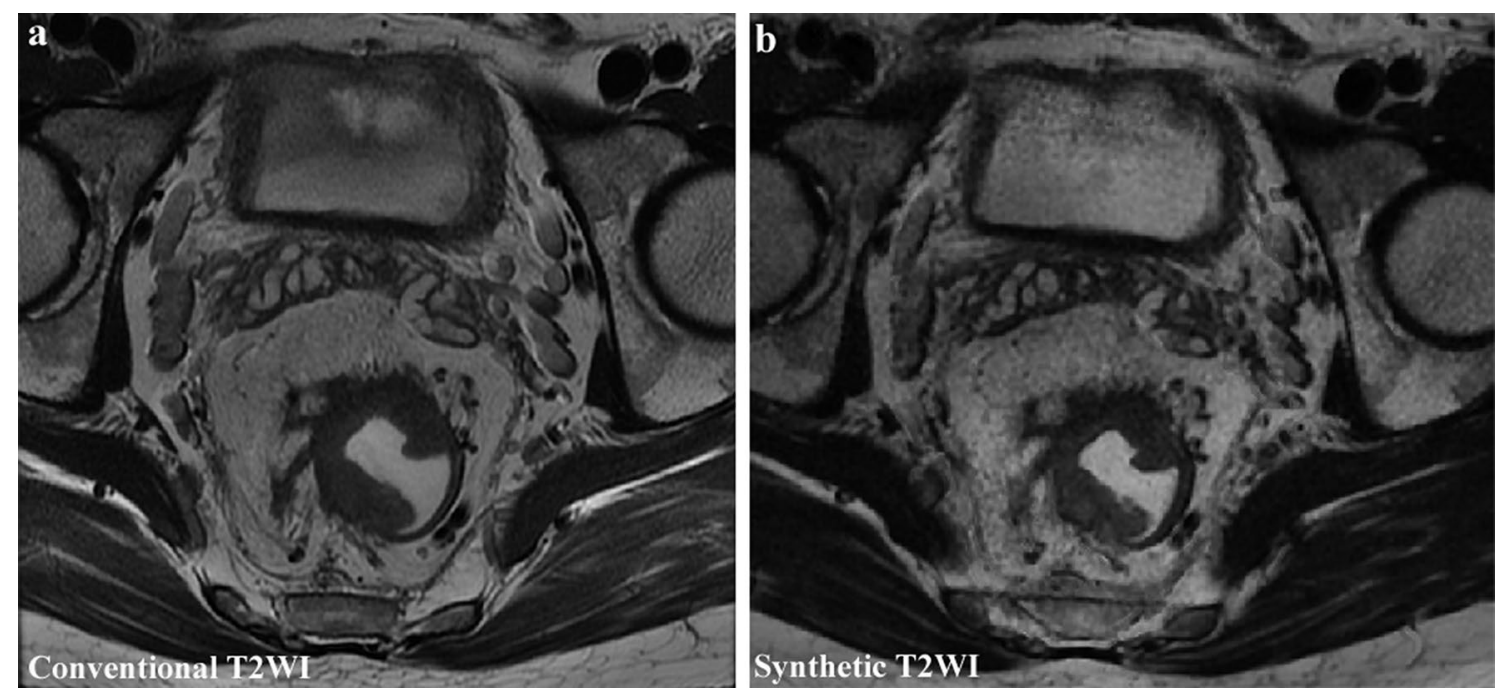

Fig. 4 Conventional and synthetic T2-weighted imaging (T2WI) from a 46-year-old male patient with rectal cancer. Synthetic T2WI provided similar image quality with the conventional T2WI. The patient was evaluated as T3 stage with extramural venous invasion using both conventional T2WI and synthetic $\mathrm{T} 2 \mathrm{Wl}$

image quality, lesion conspicuity, and the absence of motion artifacts. For the sharpness of the lesion edge, synthetic T2WI was rated superior to that of the conventional T2WI by one observer, which may be related to the observers' interpreting experience. More experienced observers may be used to conventional images. Furthermore, there was overall good to excellent interobserver agreement among the image quality scores 
Table 4 Comparison of diagnostic accuracy of T stage and EMVI using conventional and synthetic T2Wl

\begin{tabular}{llll}
\hline & Conventional T2WI & Synthetic T2WI & P value \\
\hline mrTstage & & & \\
Observer 1 & $90.4 \%(85 / 94)$ & $85.1 \%(80 / 94)$ & 0.375 \\
Observer 2 & $91.5 \%(86 / 94)$ & $87.2 \%(82 / 94)$ & 0.625 \\
mrEMVI & & & \\
Observer 1 & $81.9 \%(77 / 94)$ & $79.8 \%(75 / 94)$ & 0.625 \\
Observer 2 & $85.1 \%(80 / 94)$ & $78.7 \%(74 / 94)$ & 0.219 \\
\hline
\end{tabular}

Numbers used to calculate percentages are in parentheses. $\mathrm{mrT}$ stage, $\mathrm{T}$ stage on T2-weighted imaging; mrEMVI, extramural venous invasion on T2-weighted imaging

Table 5 Differences of quantitative parameters between the pN0 and pN1-2 groups

\begin{tabular}{llcc}
\hline Parameters & pN0 $(\mathbf{n}=\mathbf{5 6})$ & pN1-2 $(\mathbf{n}=\mathbf{2 0})$ & P value \\
\hline T1 (ms) & $1492.47(1437.33,1619.47)$ & $1492.62 \pm 173.93$ & 0.273 \\
T2 (ms) & $95.15 \pm 4.60$ & $87.44 \pm 5.66$ & $<0.001^{*}$ \\
PD (pu) & $61.45(54.65,65.91)$ & $60.44 \pm 9.017$ & 0.860 \\
\hline
\end{tabular}

Data following the normal distribution are expressed as mean \pm standard deviation. Otherwise, data are expressed as median (first quartile, third quartile) $\mathrm{PD}$, proton density ${ }^{*} p<0.05$

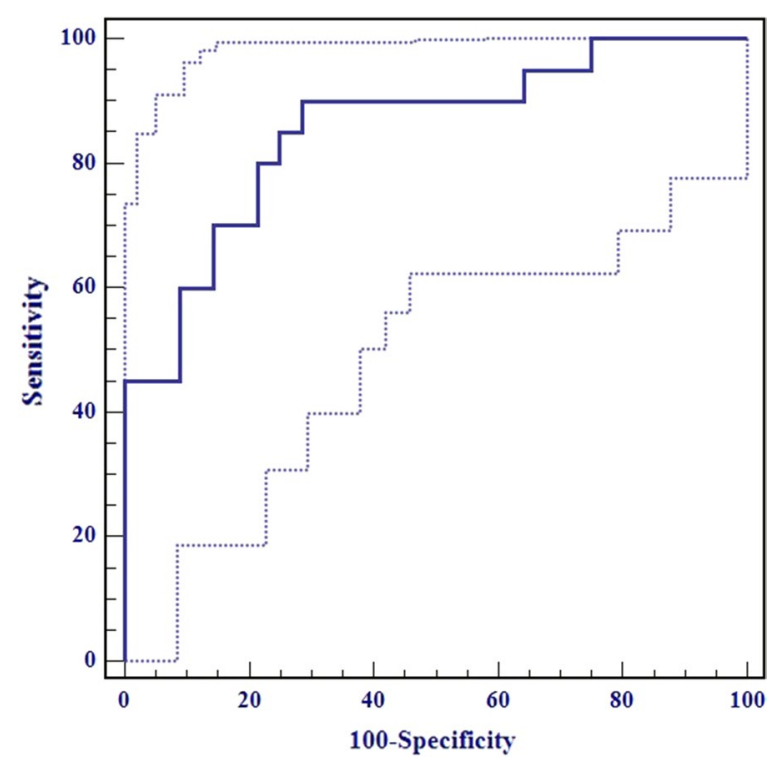

Fig. 5 Receiver operating characteristics (ROC) curve of the T2 value for predicting nodal staging of preoperatively node-negative rectal cancer with the area under the ROC, sensitivity, specificity, and accuracy of $0.854,90.0 \%, 71.4 \%$, and $80.3 \%$, respectively. The solid line represents the area under the ROC curve. The dotted line represents the $95 \%$ confidence interval for both conventional and synthetic T2WI. Therefore, we preliminarily speculated that synthetic T2WI could achieve similar image quality to that of conventional T2WI.

To further confirm the clinical feasibility of synthetic MRI, we evaluated $\mathrm{T}$ stage and EMVI using synthetic T2WI and conventional T2WI. The diagnostic accuracy of EMVI by two observers was consistent with previous studies [25-27], as was the $\mathrm{T}$ stage [28-30]. Although the diagnostic accuracy with conventional T2WI was slightly higher than that with synthetic T2WI, the difference was not statistically significant. It may be due to the fact that the signal intensity of synthetic T2WI was generally lower than that of conventional T2WI [14, 31], and observers are more accustomed to the contrast and signal intensity of conventional T2WI. Therefore, we proposed that, with training and adaptation, synthetic T2WI might be as suitable for T stage and EMVI assessment of RC as conventional T2WI.

Our previous study confirmed the value of quantitative relaxation mapping in evaluating prognostic factors of $\mathrm{RC}$, in which the potential of the T2 value for predicting $\mathrm{RC}$ nodal staging has been demonstrated [17]. Some RC patients with micro-nodal involvement may be underestimated as node-negative at initial MRI, so this study focused on these patients. A previous study established a nomogram based on clinical factors for predicting nodal staging in clinically node-negative RC patients with an AUC of 0.743 [32]. In our study, the T2 value demonstrated good diagnostic performance for predicting nodal staging of preoperatively node-negative RC with an AUC of 0.854 ( $95 \%$ CI $0.755-0.925$ ), which was superior to the previous study. We speculated that the T2 value could provide additional information for improving nodal staging of MRI-evaluated node-negative patients, which was beneficial to correctly enroll RC patients for neoadjuvant treatment.

There were some limitations in this study. Firstly, this was a single-center study with a relatively small sample size. Further prospective multi-center studies with larger sample sizes are warranted to validate our preliminary foundings. Secondly, we only evaluated the image quality of T2WI generated by synthetic MRI and did not evaluate other images. At present, high resolution T2WI is mainly used in preoperative evaluation of RC, while the application value of other non-contrasted enhanced images such as T1WI is limited. Therefore, evaluating the image quality of other contrast-weighted images is of little clinical value. Finally, we did not evaluate all tumor characteristics including sphincter invasion, and quantitative analysis of lymph node was not performed in this preliminary study. These aspects will be included in further prospective studies. 


\section{Conclusions}

Synthetic MRI may facilitate preoperative staging and EMVI evaluation of RC by providing synthetic T2WI and quantitative maps in one acquisition.

\author{
Abbreviations \\ Cl: Confidence interval; CNR: Contrast-to-noise; EMVI: Extramural venous \\ invasion; ICC: Intraclass correlation coefficient; PD: Proton density; RC: Rectal \\ cancer; ROC: Receiver operating characteristic; ROI: Regions of interest; SNR: \\ Signal-to-noise ratio; T2Wl: T2-weighted imaging.
}

\section{Supplementary Information}

The online version contains supplementary material available at https://doi. org/10.1186/s13244-021-01063-w.

Additional file 1. Interobserver agreement of imaging quality score, $\mathrm{mrT}$ stage, and mrEMVI.

\section{Authors' contributions}

LZ involved in methodology, data curation, and writing —original draft. ML involved in data curation and visualization. PW involved in software and formal analysis. YY involved in validation and investigation. $\mathrm{HZ}$ involved in supervision and writing — review and editing. XZ involved in conceptualization, supervision, and writing - review and editing. All authors read and approved the final manuscript.

\section{Funding}

There is no funding source.

\section{Availability of data and materials}

The datasets used and/or analyzed during the current study are available from the corresponding author on reasonable request.

\section{Declarations}

\section{Ethics approval and consent to participate}

This retrospective study was approved by our Institutional Review Board and the informed consent was waived.

\section{Consent for publication}

The authors of this manuscript consent for publication.

\section{Competing interests}

The authors declare that they have no competing interests.

\section{Author details}

${ }^{1}$ Department of Diagnostic Radiology, National Cancer Center/National Clinical Research Center for Cancer/Cancer Hospital, Chinese Academy of Medical Sciences and Peking Union Medical College, No. 17, Panjiayuan Nanli, Chaoyang District, Beijing 100021, China. ${ }^{2}$ GE Healthcare, MR Research China, No. 1 Tongji South Road Beijing Economic Technology Development Area, Beijing 100176, China.

Received: 10 June 2021 Accepted: 22 July 2021

Published online: 21 August 2021

\section{References}

1. Sung H, Ferlay J, Siegel RL et al (2021) Global cancer statistics 2020: GLOBOCAN estimates of incidence and mortality worldwide for 36 cancers in 185 countries. CA Cancer J Clin 71:209-249

2. Siegel RL, Miller KD, Jemal A (2020) Cancer statistics, 2020. CA Cancer J Clin 70:7-30
3. Glynne-Jones R, Wyrwicz L, Tiret E et al (2017) Rectal cancer: ESMO Clinical Practice Guidelines for diagnosis, treatment and follow-up. Ann Oncol 28:v22-v40

4. Benson AB, Venook AP, Al-Hawary MM et al (2018) Rectal cancer, version 2.2018, NCCN clinical practice guidelines in oncology. J Natl Compr Canc Netw 16:874-901

5. Li S, Liu J, Zhang F et al (2020) Novel T2 mapping for evaluating cervical cancer features by providing quantitative T2 maps and synthetic morphologic images: a preliminary study. J Magn Reson Imaging 52:1859-1869

6. Cui Y, Han S, Liu M et al (2020) Diagnosis and grading of prostate cancer by relaxation maps from synthetic MRI. J Magn Reson Imaging 52:552-564

7. Matsuda M, Kido T, Tsuda T et al (2020) Utility of synthetic MRI in predicting the Ki-67 status of oestrogen receptor-positive breast cancer: a feasibility study. Clin Radiol 75:391-398

8. Adams LC, Bressem KK, Jurmeister P et al (2019) Use of quantitative T2 mapping for the assessment of renal cell carcinomas: first results. Cancer Imaging 19:35

9. Ge YX, Hu SD, Wang Z et al (2020) Feasibility and reproducibility of T2 mapping and DWI for identifying malignant lymph nodes in rectal cancer. Eur Radiol 31:3347-3354

10. Warntjes JB, Dahlqvist O, Lundberg P (2007) Novel method for rapid, simultaneous T1, T2* ${ }^{*}$ and proton density quantification. Magn Reson Med 57:528-537

11. Warntjes JB, Leinhard OD, West J, Lundberg P (2008) Rapid magnetic resonance quantification on the brain: optimization for clinical usage. Magn Reson Med 60:320-329

12. Kumar NM, Fritz B, Stern SE, Warntjes J, Lisa CY, Fritz J (2018) Synthetic MR of the knee: phantom validation and comparison with conventional MRI. Radiology 289:465-477

13. Di Giuliano F, Minosse S, Picchi E et al (2020) Comparison between synthetic and conventional magnetic resonance imaging in patients with multiple sclerosis and controls. MAGMA 33:549-557

14. Tanenbaum LN, Tsiouris AJ, Johnson AN et al (2017) Synthetic MRI for clinical neuroimaging: results of the magnetic resonance image compilation (MAGiC) prospective, multicenter, multireader trial. AJNR Am J Neuroradiol 38:1103-1110

15. Matsuda M, Tsuda T, Ebihara R et al (2021) Enhanced masses on contrastenhanced breast: differentiation using a combination of dynamic contrast-enhanced MRI and quantitative evaluation with synthetic MRI. J Magn Reson Imaging 53:381-391

16. Arita Y, Takahara T, Yoshida S et al (2019) Quantitative assessment of bone metastasis in prostate cancer using synthetic magnetic resonance imaging. Invest Radiol 54:638-644

17. Zhao L, Liang M, Xie L, Yang Y, Zhang H, Zhao X (2021) Prediction of pathological prognostic factors of rectal cancer by relaxation maps from synthetic magnetic resonance imaging. Eur J Radiol 138:109658

18. Zhao L, Liang M, Wang S, Yang Y, Zhang H, Zhao X (2021) Preoperative evaluation of extramural venous invasion in rectal cancer using radiomics analysis of relaxation maps from synthetic MRI. Abdom Radiol (NY). https://doi.org/10.1007/s00261-021-03021-y

19. Li XT, Sun YS, Tang L, Cao K, Zhang XY (2015) Evaluating local lymph node metastasis with magnetic resonance imaging, endoluminal ultrasound and computed tomography in rectal cancer: a meta-analysis. Colorectal Dis 17:0129-0135

20. Weiser MR (2018) AJCC 8th edition: colorectal cancer. Ann Surg Oncol 25:1454-1455

21. He M, Xu J, Sun Z et al (2020) Prospective comparison of reduced fieldof-view (rFOV) and full FOV (fFOV) diffusion-weighted imaging (DWI) in the assessment of insulinoma: image quality and lesion detection. Acad Radiol 27:1572-1579

22. Smith NJ, Barbachano Y, Norman AR, Swift RI, Abulafi AM, Brown G (2008) Prognostic significance of magnetic resonance imaging-detected extramural vascular invasion in rectal cancer. Br J Surg 95:229-236

23. Beets-Tan R, Lambregts D, Maas M et al (2018) Magnetic resonance imaging for clinical management of rectal cancer: updated recommendations from the 2016 European Society of Gastrointestinal and Abdominal Radiology (ESGAR) consensus meeting. Eur Radiol 28:1465-1475

24. Jung Y, Gho SM, Back SN, Ha T, Kang DK, Kim TH (2018) The feasibility of synthetic MRI in breast cancer patients: comparison of T(2) relaxation 
time with multiecho spin echo T(2) mapping method. Br J Radiol 92:20180479

25. Kim TH, Woo S, Han S, Suh CH, Vargas HA (2019) The Diagnostic performance of MRI for detection of extramural venous invasion in colorectal cancer: a systematic review and meta-analysis of the literature. AJR Am J Roentgenol 213:575-585

26. Bae JS, Kim SH, Hur BY et al (2019) Prognostic value of MRI in assessing extramural venous invasion in rectal cancer: multi-readers' diagnostic performance. Eur Radiol 29:4379-4388

27. Ale AH, Kirsch R, Razaz S et al (2019) Extramural venous invasion in recta cancer: overview of imaging, histopathology, and clinical implications. Abdom Radiol (NY) 44:1-10

28. Xu L, Zhang Z, Qin Q, Zhang C, Sun X (2020) Assessment of T and N staging with MRI(3)T in lower and middle rectal cancer and impact on clinical strategy. J Int Med Res 48:1220728237
29. Lu ZH, Hu CH, Qian WX, Cao WH (2016) Preoperative diffusion-weighted imaging value of rectal cancer: preoperative T staging and correlations with histological T stage. Clin Imaging 40:563-568

30. Xu L, Zhang C, Zhang Z, Qin Q, Sun X (2020) Value of 3Tesla MRI in the preoperative staging of mid-low rectal cancer and its impact on clinical strategies. Asia Pac J Clin Oncol 16:e216-e222

31. Drake-Pérez M, Delattre B, Boto J et al (2018) Normal values of magnetic relaxation parameters of spine components with the synthetic MRI sequence. AJNR Am J Neuroradiol 39:788-795

32. Zhou C, Liu HS, Liu XH et al (2019) Preoperative assessment of lymph node metastasis in clinically node-negative rectal cancer patients based on a nomogram consisting of five clinical factors. Ann Transl Med 7:543

\section{Publisher's Note}

Springer Nature remains neutral with regard to jurisdictional claims in published maps and institutional affiliations.

\section{Submit your manuscript to a SpringerOpen ${ }^{\circ}$ journal and benefit from:}

- Convenient online submission

- Rigorous peer review

- Open access: articles freely available online

- High visibility within the field

- Retaining the copyright to your article

Submit your next manuscript at $\boldsymbol{\nabla}$ springeropen.com 